\title{
The MIQE Revolution: Implementation of standards for the reporting of quantitative PCR studies
}

\author{
Duncan Ayers 1, 2 \\ ${ }^{1}$ Department of Pathology, Faculty of Medicine \& Surgery, University of Malta, Msida, Malta. \\ ${ }^{2}$ Faculty of Medical \& Human Sciences, The University of Manchester, Manchester, UK.
}

Received January 16, 2014; Revised March 10, 2014; Accepted March 10, 2014; Published Online April 9, 2014

\section{Scientific $\mathcal{N o t e}$}

\begin{abstract}
The discovery of the polymerase chain reaction (PCR) a few decades ago initiated a global impact on the entirety of the medical and life sciences research spheres. Nowadays, essentially all laboratories focusing on such vital research employ in-house PCR techniques on a near-daily basis, due to the wide spectrum of applications which PCR technology can adopt itself to. Unfortunately, ubiquitously available and affordable technologies, such as RT-qPCR, do have a major passive drawback: inter-laboratory reproducibility. Variations in the routine methodologies implemented by individual laboratories can inevitably lead to severe lapse of data robustness and reliability for publication in peer-reviewed journals. In order to address this pressing issue, a consortium of eminent research group leaders in the field of RT-qPCR technology decided to propose a distinct set of standardized guidelines for the reporting of RT-qPCR study results, known as the Minimum Information for Publication of Quantitative Real Time PCR Experiments (MIQE), which were published in early 2009. This concept is very much similar to the one leading to the development of the Minimum Information for Microarray Experimets (MIAME) guidelines for reporting of microarray-based studies. In order to address this pressing issue, a consortium of eminent research group leaders in the field of RT-qPCR technology decided to propose a distinct set of standardized guidelines for the reporting of RT-qPCR study results, known as the Minimum Information for Publication of Quantitative Real Time PCR Experiments (MIQE), which were published in early 2009. This concept is very much similar to the one leading to the development of the Minimum Information for Microarray Experimets (MIAME) guidelines for reporting of microarray-based studies.
\end{abstract}

Keywords: qPCR; MIQE; Guidelines; Standardisation

The discovery of the polymerase chain reaction (PCR) a few decades ago initiated a global impact on the entirety of the medical and life sciences research spheres. Nowadays, essentially all laboratories focusing on such vital research employ in-house PCR techniques on a near-daily basis, due to the wide spectrum of applications which PCR technology can adopt itself to, such as cloning and medical diagnostics to name but a few. In addition, the cost for purchasing and maintenance of basic PCR equipment such as thermal cyclers and real time PCR platforms is within reach of most laboratories with adequate research funds.

\footnotetext{
Corresponding author: Duncan Ayers; Department of Pathology, Faculty of Medicine \& Surgery, University of Malta, Msida MSD2080, Malta.

Cite this article as:

Ayers D. The MIQE Revolution: Implementation of standards for the reporting of quantitative PCR studies. Int J Cancer Ther Oncol 2014; 2(2):02026. DOI: 10.14319/ijcto.0202.6
}

The combined sensitivity, reproducibility and cost-effectiveness of PCR technology consequently allowed for the refined development for applications in the fields of quantitative genomics and transcriptomics analytical methods, such as gene expression analyses. Furthermore, the emergence of novel non-coding RNA molecular families, such as micro RNAs (miRNAs) and long non-coding RNAs (lncRNAs), as novel key regulators of gene expression only serve to augment the importance of quantitative PCR technology as a powerful research tool within the context of such research fields. A typical example of such applications is the utilization of high throughput reverse transcription quantitative PCR (RT-qPCR) for whole genome miRNA expression profiling. ${ }^{1}$

Unfortunately, ubiquitously available and affordable technologies, such as RT-qPCR, do have a major passive drawback: inter-laboratory reproducibility. Variations in the routine methodologies implemented by individual laboratories can inevitably lead to severe lapse of data robustness and 
reliability for publication in peer-reviewed journals. These variations can occur at differing stages of the RT-qPCR procedure include the stringency levels involved in target gene primer assay designs, quality of handling and/or storage of clinical RNA samples, efficiency of RNA extraction and/or reverse transcription protocols, and normalization methods utilized for relative quantification of RT-qPCR data obtained post-run. Consequently, such a lack of standardization regarding essential methodologies and practices pertaining to the RT-qPCR process ultimately leads to the production of non-reproducible data. This obtained data can, essentially, be of no concrete value and should therefore not be deemed fit for publication by scientific journals. However, the lack of clear standards and guidelines can easily allow such situations to take place, possibly within well -respected scientific journals as well.

In order to address this pressing issue, a consortium of eminent research group leaders in the field of RT-qPCR technology decided to propose a distinct set of standardized guidelines for the reporting of RT-qPCR study results, known as the Minimum Information for Publication of Quantitative Real Time PCR Experiments (MIQE), which were published in early 2009. ${ }^{2}$ This concept is very much similar to the one leading to the development of the Minimum Information for Microarray Experimets (MIAME) guidelines for reporting of microarray-based studies. ${ }^{3}$

The MIQE guidelines consist of a 'checklist' of essential and desirable information parameters relating to each individual step of the RT-qPCR experimental procedure. ${ }^{2}$ Highlights of these guidelines include the importance of reporting all parameters required for the design of efficient primer sets for each individual target and reference gene investigated in any particular study.

This includes (but not limited to) the reporting of the full primer sequences, target gene accession number, target gene region (ie. Whether intron spanning or otherwise, splice variant possibilities, etc.), secondary structure formations of the individual primers and target template sequence, primer set homology search and target template region single nucleotide polymorphism (SNP) / other mutation check methodologies. The development of individual standard curves for each individual primer set utilized in the RT-qPCR study is also required to confirm the dynamic range and efficiency of the primer set to allow for a successful PCR run. Any primer sets that fail to produce efficient standard curves should be removed from utilization in the study and new primer sets should be designed accordingly.

The MIQE guidelines also stress the importance of ensuring that clinical samples and consequent extracted RNA are to be handled and stored in the appropriate manner. Furthermore, researchers should include parameters such as the level of
RNA purity and integrity for each individual clinical sample utilized in their RT-qPCR study.

Other major parameters that require to be reported include the RNA input amounts utilized in the reverse transcription step, type of reverse transcription enzyme and the priming method employed for such measure. The necessity to implement more than just one reference gene (and with prior validation) for the relative quantification RT-qPCR technique is also clearly stressed within the MIQE guidelines.

Ultimately, this checklist should be utilized as a fixed referral point for all researchers embarking on RT-qPCR research studies, in order to ensure that all their RT-qPCR protocols adhere to the guidelines present on the MIQE checklist prior to submission of research results for publication. Similarly, the recent emergence of digital qPCR technology has motivated the MIQE consortium to develop an publish the digital MIQE guidelines for proper utilization and scientific reporting of research studies employing this novel technology. ${ }^{4}$

Leading members of the MIQE consortium recently published in Nature Methods the results of a survey in over 1700 RT-qPCR publications, examining the level of adherence by researchers to the MIQE guidelines when reporting such studies. $^{5}$ The results of this survey revealed that for RT-qPCR studies published between 2009 and 2011, overall adherence to MIQE guidelines was reduced particularly in publications from scientific journals having an Impact Factor of 10 or higher. ${ }^{5}$ For studies published between 2012 and 2013, the levels of reporting qPCR study results in compliance with the MIQE standards was increased. ${ }^{5}$ Sadly, this was not the case for publications from leading, very high impact factor journals during the same time period. ${ }^{5}$

In conclusion, the widespread accessibility to qPCR technology has allowed for its own abuse due to the ongoing reporting of poorly conceived qPCR datasets through inappropriate and/or lack of standards in the various stages of the qPCR experimental design and procedure. More alarming is the fact that, particularly for high Impact Factor scientific journals, there is a pressing urge to apply added scrutiny by the journal-appointed peer-reviewers, of scientific manuscripts bearing clinical / research affirmations based on qPCR data findings, prior to their publication.

Fortunately, since its inception in 2009, the concept of standardization and streamlined reporting of $\mathrm{qPCR}$ data through dissemination of the MIQE guidelines is gradually seeping into the minds of researchers. This consequently ensures that precious research efforts can be deemed to translate into a robust piece of scientific evidence having reliable clinical value, and upon which other peer researchers can build on. Time will tell about the long-term effects of the MIQE revolution, though at present, its voice is already being heard. 


\section{Conflict of interest}

The authors declare that they have no conflicts of interest. The authors alone are responsible for the content and writing of the paper.

\section{References}

1. Mestdagh $\mathrm{P}$, Derveaux $\mathrm{S}$, Vandesompele J. Whole-genome RT-qPCR microRNA expression profiling. Methods Mol Biol 2012; 815:121- 30.

2. Bustin SA, Benes V, Garson JA, et al. The MIQE guidelines: minimum information for publication of quantitative real-time PCR experiments. Clin Chem 2009; 55:611-22.

3. Brazma A, Hingamp P, Quackenbush J, et al. Minimum information about a microarray experiment (MIAME)-toward standards for microarray data. Nat Genet 2001; 29:365-71.

4. Huggett JF, Foy CA, Benes V, Emslie K, et al. The digital MIQE guidelines: Minimum Information for Publication of Quantitative Digital PCR Experiments. Clin Chem 2013; 59:892-902.

5. Bustin SA, Benes V, Garson J, et al. The need for transparency and good practices in the qPCR literature. Nat Methods 2013; 10:1063-7. 